\title{
CORRECTION
}

\section{Correction to: Cannabis use is associated with patient and clinical factors in a population-based sample of colorectal cancer survivors}

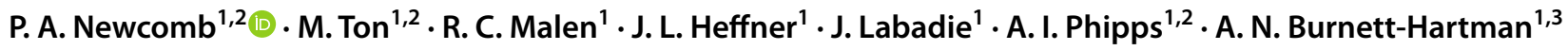

Published online: 19 October 2021

(c) Springer Nature Switzerland AG 2021

\section{Correction to: Cancer Causes \& Control \\ https://doi.org/10.1007/s10552-021-01468-4}

Following publication of the original article, the author has identified an error in Table 1.

The correct version of Table 1 is given below.

The original article has been revised.

The original article can be found online at https://doi.org/10.1007/ s10552-021-01468-4.

P. A. Newcomb

newcomb@fredhutch.org

1 Public Health Sciences Division, Fred Hutchinson Cancer Research Center, 1100 Fairview Avenue N, Seattle, WA 98109, USA

2 Department of Epidemiology, University of Washington, Seattle, WA, USA

3 Institute for Health Research, Kaiser Permanente Colorado, Denver, CO, USA 
Table 1 Odds ratios comparing current cannabis use among CRC patients in the Puget Sound region of Washington State according to participant characteristics, clinical factors, and quality of life (FACT-C) scores $(n=1,433)$

\begin{tabular}{|c|c|c|c|c|}
\hline & $\begin{array}{l}\text { Never/Former Can- } \\
\text { nabis Users } \\
n=1,094 \\
n(\%)\end{array}$ & $\begin{array}{l}\text { Current Can- } \\
\text { nabis Users } \\
n=339 \\
n(\%)\end{array}$ & Unadjusted OR (95\% CI) & Adjusted* OR (95\% CI) \\
\hline \multicolumn{5}{|l|}{ Age (years) } \\
\hline $65+$ & $374(34.2)$ & $66(19.5)$ & 1.00 (ref) & 1.00 (ref) \\
\hline $55-64$ & $329(30.1)$ & $118(34.8)$ & $1.75(1.26,2.43)$ & $1.85(1.30,2.62)$ \\
\hline $45-54$ & $286(26.1)$ & $103(30.4)$ & $1.96(1.40,2.75)$ & $2.20(1.53,3.17)$ \\
\hline $18-44$ & $105(9.6)$ & $52(15.3)$ & $2.86(1.87,4.37)$ & $3.30(2.07,5.26)$ \\
\hline \multicolumn{5}{|l|}{ Sex } \\
\hline Female & $514(47.0)$ & $139(41.0)$ & 1.00 (ref) & 1.00 (ref) \\
\hline Male & $580(53.0)$ & $200(59.0)$ & $1.28(1.00,1.63)$ & $1.17(0.89,1.55)$ \\
\hline \multicolumn{5}{|l|}{ Race } \\
\hline Non-Hispanic White & $854(78.1)$ & $275(81.1)$ & 1.00 (ref) & 1.00 (ref) \\
\hline Black & $31(2.8)$ & $15(4.4)$ & $1.50(0.80,2.83)$ & $1.53(0.76,3.07)$ \\
\hline Hispanic & $24(2.2)$ & $8(2.4)$ & $1.04(0.46,2.33)$ & $0.77(0.32,1.86)$ \\
\hline Asian \& Pacific Islander & $84(7.7)$ & $10(3.0)$ & $0.37(0.19,0.72)$ & $0.29(0.14,0.58)$ \\
\hline Other & $58(5.3)$ & $18(5.3)$ & $0.96(0.56,1.66)$ & $0.77(0.43,1.38)$ \\
\hline \multicolumn{5}{|l|}{ Education } \\
\hline College graduate or higher & $505(46.2)$ & $138(40.7)$ & 1.00 (ref) & 1.00 (ref) \\
\hline Some College & $374(34.2)$ & $114(33.6)$ & $1.11(0.84,1.47)$ & $1.10(0.81,1.51)$ \\
\hline HS graduate or less & $205(18.7)$ & $84(24.8)$ & $1.49(1.09,2.05)$ & $1.35(0.95,1.92)$ \\
\hline \multicolumn{5}{|l|}{ BMI } \\
\hline$<25.0$ & $365(33.4)$ & $155(45.7)$ & 1.00 (ref) & 1.00 (ref) \\
\hline $25.0-29.9$ & $340(31.1)$ & 97 (28.6) & $0.70(0.52,0.94)$ & $0.65(0.48,0.90)$ \\
\hline $30.0+$ & $359(32.8)$ & $81(23.9)$ & $0.55(0.41,0.75)$ & $0.49(0.35,0.68)$ \\
\hline \multicolumn{5}{|l|}{ Smoking } \\
\hline Never & $647(59.1)$ & $151(44.5)$ & 1.00 (ref) & 1.00 (ref) \\
\hline Former & $359(32.8)$ & $145(42.8)$ & $1.72(1.33,2.24)$ & $1.90(1.42,2.54)$ \\
\hline Current & $83(7.6)$ & $41(12.1)$ & $2.11(1.39,3.18)$ & $1.82(1.15,2.88)$ \\
\hline \multicolumn{5}{|l|}{ Alcohol } \\
\hline Non-/Occasional Drinkers (<1 g/day) & $576(52.7)$ & $132(38.9)$ & 1.00 (ref) & 1.00 (ref) \\
\hline Light-to-Moderate Drinkers (1-28 g/day) & $394(36.0)$ & $138(40.7)$ & $1.50(1.15,1.96)$ & $1.36(1.01,1.83)$ \\
\hline Heavy Drinkers ( $>28$ g/day) & $109(10.0)$ & $63(18.6)$ & $2.48(1.73,3.55)$ & $1.97(1.32,2.96)$ \\
\hline \multicolumn{5}{|l|}{ FACT-C } \\
\hline Lowest quartile & $230(21.0)$ & $114(33.6)$ & $1.91(1.46,2.51)$ & $1.51(1.12,2.02)$ \\
\hline \multicolumn{5}{|l|}{ Stage } \\
\hline Localized & $447(40.9)$ & $75(22.1)$ & 1.00 (ref) & 1.00 (ref) \\
\hline Regional & 455 (41.6) & $162(47.8)$ & $2.08(1.54,2.79)$ & $2.11(1.54,2.90)$ \\
\hline Distant & $167(15.3)$ & $96(28.3)$ & $3.35(2.37,4.73)$ & $3.12(2.15,4.52)$ \\
\hline \multicolumn{5}{|l|}{ Site } \\
\hline Proximal & $359(32.8)$ & $101(29.8)$ & 1.00 (ref) & 1.00 (ref) \\
\hline Distal & $309(28.2)$ & 107 (31.6) & $1.26(0.92,1.71)$ & $1.11(0.79,1.56)$ \\
\hline Rectal & $408(37.3)$ & $128(37.8)$ & $1.14(0.85,1.53)$ & $0.84(0.60,1.16)$ \\
\hline \multicolumn{5}{|l|}{ Ostomy } \\
\hline No Ostomy & $887(81.1)$ & $252(74.3)$ & 1.00 (ref) & 1.00 (ref) \\
\hline Ostomy & 203 (18.6) & $86(25.4)$ & $1.49(1.12,1.99)$ & $1.09(0.80,1.49)$ \\
\hline
\end{tabular}

*Adjusted for age, sex, race, BMI, smoking status, alcohol, education, and tumor stage 
Publisher's Note Springer Nature remains neutral with regard to jurisdictional claims in published maps and institutional affiliations. 